\title{
Biomass distances from personalized multispecies dynamic flux balance analysis of the human gut microbiome identify dietary influences for patients with and without inflammatory bowel disease
}

\author{
Alexandros Dimopoulos ${ }^{1,2}$, Martin Reczko ${ }^{2 *}$ \\ ${ }^{1}$ Institute of Chemical Engineering Sciences, Foundation for Research and Technology Hellas, Patras, 26504, Greece, \\ ${ }^{2}$ Institute for Fundamental Biomedical Science, Biomedical Sciences Research Center "Alexander Fleming", Vari, 16672, Greece \\ *Correspondence to: reczko@fleming.gr
}

\begin{abstract}
A parallelized version of a multispecies dynamic flux balance analysis (msdFBA) algorithm is implemented and applied to the AGORA collection of genome-scale metabolic reconstructions for 818 members of the human gut microbiome. The msdFBA method assumes the well stirred interaction mode of all organisms to exchange external metabolites. In each msdFBA simulation, the biomasses the gut microbiome composition of one of 149 patients from NIH Human Microbiome Project is used for initialization in combination with one of 11 different diets used as substrates as defined in the Virtual Metabolic Human database. The union of all species in the patient data comprises 255 different microbes. The patients are either healthy or suffer from inflammatory bowel disease (IBD). The msdFBA simulation is performed for 50 time steps. For all combinations of patients and time steps, the euclidean distance between the vector of the biomasses of the 255 patient species and the evolving vector of biomasses for the same species is calculated, providing the information about the biomass distance to each patient during each simulation. To quantify the overall influence of a diet for all patients, a diet score is defined as the sum of the reciprocal distances to the closest patient at the last time step, in case the closest patient is diseased, subtracted from the respective sum for the case that the closest patient is healthy. With this score, the known beneficial influences both of a high fiber and a gluten free diet for IBD is verified. Noteworthy is the utility of a Mediterranean diet in this context, having similar distance patterns. The proposed method provides an universal platform for the in-silico analysis of different environmental influences like diets for different microbiotas defined by metagenomic quantifications from individual patients and has the potential to generate additional dietary recommendations for the management of various other diseases.
\end{abstract}

\section{INTRODUCTION}

A large bacterial community resides in the human gut, acting as a pathogen defense and supporting the metabolism of indigestible food compounds among many other functions [1]. Deviations of the abundance of specific species contribute to various diseases such as inflammatory bowel disease (IBD) [1]. We introduce an in-silico method to assess environmental influences such as diet on the dynamic individual gut microbiome composition for a patient via measurements of the compositional distance to other patients with known health status.

\section{MATERIALS AND METHODS}

\section{Diet substrates}

The used diet substrate constraints are defined in the Virtual Metabolic Human database [2] and are characterized as Vegetarian: ovo-lacto-vegetarian diet; Vegan: without animal derived products; AverageEuropean: designed after nutrient-based survey for European countries; Mediterranean: composed of an abundance of fresh plant foods, minimally processed food, olive oil as the principal fat source, dairy products are consumed daily, poultry and fish are consumed in a moderate amount and red meat is consumed in a very low amount; DACH: based on the recommended amount of nutrients 
established by the society for Nutrition in Switzerland Germany and Austria; HighProtein: typical diet for strength athletes or often prescribed for overweight people for weight loss; GlutenFree: based on a 24 hour weight protocol from a subject who suffers from gluten intolerance; HighFiber: contains animal derived products and exceeds the fiber amount of the vegan diet; Type 2 diabetes: high amounts of vitamins and minerals with antioxidative capacities, such as vitamin $\mathrm{C}$ and $\mathrm{E}$, low kcal amount; HighFatLowCarb: imitates a ketogenic diet. Composed of 1,7\% of energy of carbohydrates, $70 \%$ of energy of lipids and 24\% of energy of proteins; Unhealthy: designed to contain a very low amount of dietary fibers, high kcal amount, simple sugars, saturated fatty acids, and cholesterol.

\section{Gut microbiome patient data and gut microbiome genome-scale metabolic reconstructions}

The gut microbiome composition of 149 patients from NIH Human Microbiome Project available at [3] is used. The data contains 5 healthy and 144 patients with IBD. The AGORA collection of genomescale metabolic reconstructions, version 1.03 containing 818 species is used [4].

\section{Multispecies dynamic flux balance analysis}

The multispecies dynamic flux balance analysis (msdFBA) algorithm described in [5] has been modified to support parallel execution of all linear programming optimizations. To allow for species with decaying biomasses, a small constant of 0.017 called endogenousDecayRate is subtracted from all obtained growth rates. This is the minimal growth rate measured for a the first step of a msdFBA over all diets and all patients. Before each simulation, the biomasses are initialized to the patient specific abundances. To allow the growth of initially non-present species, the biomass of each absent species is initialized to $1 / 100$ th of the smallest species biomass occurring in all patients. Each simulation is run for 50 time steps.

\section{Diet score}

The overall influence of a diet to the health status of a patient is quantified using:

$$
\text { Diet }_{\text {score }}=\frac{1}{n_{\text {patients }}} \sum_{\text {patient }} \frac{\text { closest }_{\text {healthy }}}{1+d_{\text {closest }}}
$$

where $d_{\text {closest }}$ is the closest biomass distance to any of the $n_{\text {patients }}=149$ patients at the final time step of a msdFBA simulation and

$$
\text { closest }_{\text {healthy }}=\left\{\begin{array}{c}
+1, \text { if closest patient is healthy, } \\
-1, \text { else }
\end{array}\right.
$$

If all patients move their biomass composition to one of the healthy patients during the simulation of the diet, the Diet $_{\text {score }}$ is 1 . If the closest patient at the final step was always diseased, Diet score is 1 . 


\section{Comparison of diet scores}

As a Shapiro test confirms the non-normality of the scores for each diet over all patients, a Friedman test [6] is performed to test the occurrence of a different distribution in any of the diets, given the diets can be treated as repeated measurements for each patient. Post hoc assessment of differences between the diets is performed using Wilcoxon-Nemenyi-McDonald-Thompson tests [7] as implemented by [8] and the negative log p-values of this test is used as a distance measure between the diets.

\section{RESULTS}

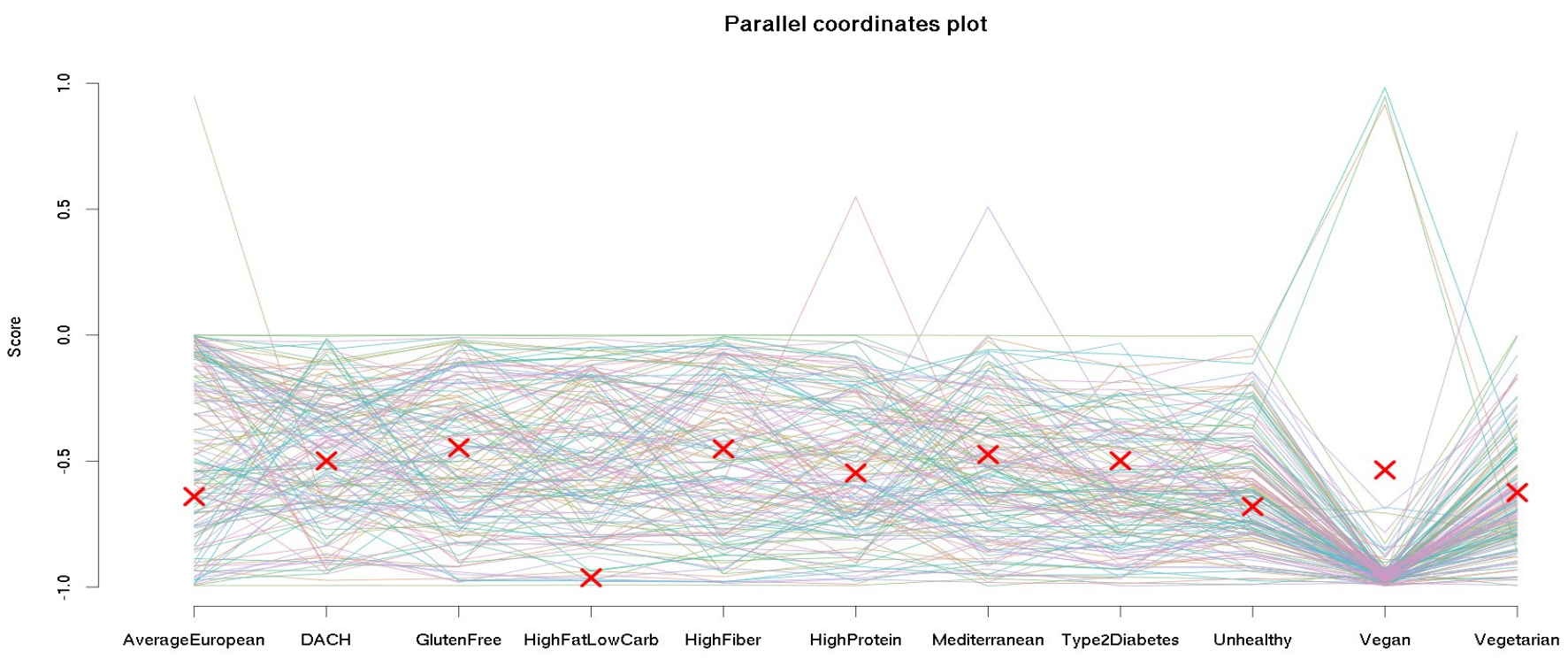

Figure 1. Contribution of each patient to Diet $_{\text {score }}$ for IBD.

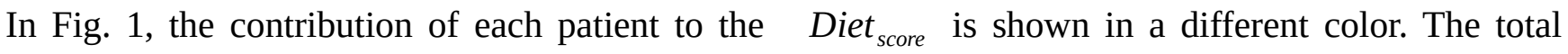
Diet $_{\text {score }}$ for each diet is marked with crosses. The three highest scoring diets are: HighFiber, GlutenFree and Mediterranean, reproducing known observations [9]. The personalized character of this analysis is evident not only in the case of the Vegan diet, which would direct three patients exactly to a healthy biomass composition, while the rest of the patients do not show evidence of a benefit.

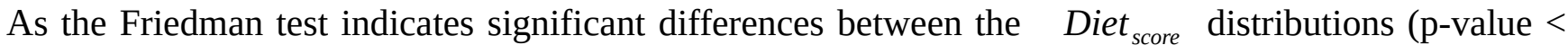
2.2e-16), the distances between the diets defined by the post hoc tests are used to project the diets in two dimensions using multidimensional scaling shown in Fig. 2. The diets can be grouped into three clusters indicated with colored boxes, where the post hoc tests to all diets outside of a cluster show significant differences ( $p$-value $<0.05$ ), while the tests for all diet pairs within a cluster do not indicate significant differences. The only marginal exception is the test between the Vegetarian and the Vegan diet with a p-value of 0.0846 . This averaged analysis of the relations between the diets is useful for some general recommendations, however the major contribution of the method presented is the 
generation of the time courses of personalized profiles in Fig. 1 that can be easily applied to analyze environmental influences in a collection of other diseases related to individual microbiotas.

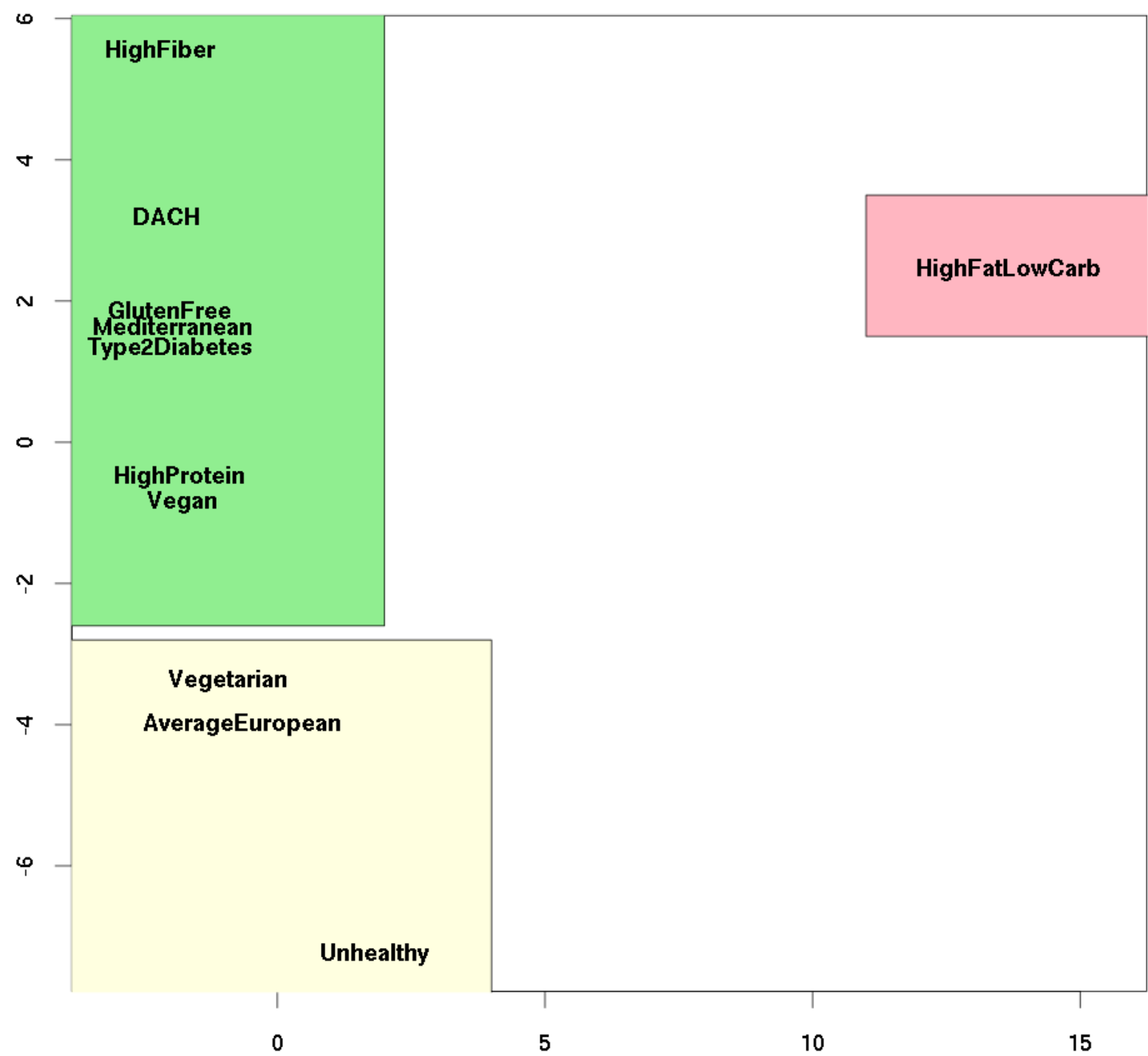

Figure 2. MDS projection of the $\quad$ Diet $_{\text {score }}$ distances for IBD. The color of the three clusters of diets indicate the utility of the diet for IBD
(green/yellow/red: top/middle/lower Diet $_{\text {score }}$ group).

\section{ACKNOWLEDGEMENTS}

AD has been supported for this work by the "ELIXIR-GR: Managing and Analysing Life Sciences Data" (MIS: 5002780) project that is co-financed by Greece and the European Union - European Regional Development Fund. 


\section{REFERENCES}

[1] F. Guarner and J. Malagelada, "Gut flora in health and disease”, The Lancet, 361 (9356): 512-19, 2003.

[2] A. Noronha,J. Modamio, Y. Jarosz, E. Guerard, N. Sompairac, G. Preciat et al. “The Virtual Metabolic Human database: integrating human and gut microbiome metabolism with nutrition and disease”, Nucleic Acids Res, 47:D614-24, 2019.

[3] F. Baldini, A. Heinken, L. Heirendt, S. Magnusdottir, RMT. Fleming, I. Thiele, "The Microbiome Modeling Toolbox: from microbial interactions to personalized microbial communities", Bioinformatics, 35(13):2332-2334, 2019.

[4] S. Magnusdottir, A. Heinken, L. Kutt, DA. Ravcheev, E. Bauer, A. Noronha et al. Generation of genome-scale metabolic reconstructions for 773 members of the human gut microbiota. Nat Biotechnol, 35:81-9, 2017

[5] E. Tzamali, P. Poirazi, I. Tollis and M. Reczko, “A computational exploration of bacterial metabolic diversity identifying metabolic interactions and growth-efficient strain communities”, BMC Systems Biology, 5:167, 2011.

[6] M. Friedman, “A comparison of alternative tests of significance for the problem of m rankings”, The Annals of Mathematical Statistics, 11 (1): 86-92, 1940.

[7] M. Hollander and D.A. Wolfe, Nonparametric Statistical Methods, 2nd Edition. New York: John Wiley \& Sons, 1999, p. 295.

[8] T. Galili, "Post hoc analysis for Friedman's Test”, https://www.r-statistics.com/2010/02/post-hocanalysis-for-friedmans-test-r-code , 2010.

[9] MI. Vazquez-Roque, M. Camilleri, T. Smyrk, JA. Murray, E. Marietta, J. O’Neill et al. “A controlled trial of gluten-free diet in patients with irritable bowel syndrome-diarrhea: effects on bowel frequency and intestinal function”, Gastroenterolog, 144:903-911.e3, 2013 